\title{
Investigation of Different Pre-Treated Multi-Walled Carbon Nanotubes by Raman Spectroscopy
}

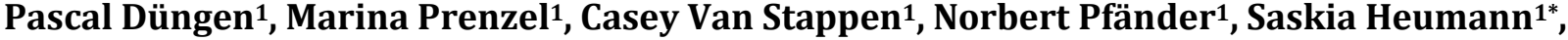 Robert Schlögl1,2}

${ }^{1}$ Max Planck Institute for Chemical Energy Conversion, Mülheim an der Ruhr, Germany

${ }^{2}$ Fritz Haber Institute of the Max Planck Society, Berlin, Germany

Email: *saskia.heumann@cec.mpg.de

How to cite this paper: Düngen, P., Prenzel, M., Van Stappen, C., Pfänder, N., Heumann, S. and Schlögl, R. (2017) Investigation of Different Pre-Treated Multi-Walled Carbon Nanotubes by Raman Spectroscopy. Materials Sciences and Applications, 8, 628641.

https://doi.org/10.4236/msa.2017.88044

Received: June 14, 2017

Accepted: August 14, 2017

Published: August 17, 2017

Copyright $\odot 2017$ by authors and Scientific Research Publishing Inc. This work is licensed under the Creative Commons Attribution International License (CC BY 4.0).

http://creativecommons.org/licenses/by/4.0/

\begin{abstract}
Raman spectroscopy is a common method of studying carbon-based materials such as multi-walled carbon nanotubes (MWCNT). However, the analysis of this technique is non-trivial since recorded spectra may be a convolution of both molecular vibrations and phonon resonances. The energies of these physical processes may occur in the same energy regime, and hence several analytical approaches can be necessary for a full analysis. Due to the negligible quantities of non-graphitic carbon in MWCNT, the present fitting procedure focuses on understanding phonon resonances to elucidate how varying modifications of MWCNT ultimately influence their graphitic bulk structure. We have found this approach to provide greater insight into the structure of MWCNT when low quantities of amorphous carbon are present, when compared with methods which try to interpret both phonon scattering and molecular vibrations simultaneously. Different pre-treatments for the modification of the graphitic structure of MWCNT are compared, including aqueous acidic and gas phase methods, and statistically evaluated. Focusing on phonon resonances enables one to analyze the reaction process of nitrosulfuric acid pre-treatment at different temperatures. Thereby, it is possible to control the ratio between defects and graphitic structures in MWCNT samples and prepare samples with reproducible D/G ratios.
\end{abstract}

\section{Keywords}

CNT, Raman, Total Reflection X-Ray Fluorescence, Raman Spectra Fitting, CNT Functionalization, Metal Contamination 


\section{Introduction}

Multi-walled carbon nanotubes (MWCNT) are highly interesting materials with many applications in catalysis, electronics, and the bio/medical fields due to their unique electrical and mechanical properties [1]. However, MWCNT do not exhibit uniform structures, and therefore, require cleaning processes in order to guarantee a uniform base material prior to any modification or direct application [2]. In this work, we use co-axial structured MWCNT composed of monoatomic sheets of polyaromatic $\mathrm{sp}^{2}$ hybridized carbon layers (equivalent of graphene) which are rolled into cylinders to form highly entangled networks [3]. The ends of these cylinders are closed with caps in the form of a half sphere of fullerene. These structures exhibit high surface areas $\left(250 \mathrm{~m}^{2} / \mathrm{g}\right)$ and conductivities which are comparable to classic carbon materials such as graphite [4]. Due to their high surface area, MWCNT are often used in catalysis as supports for catalytically active species. Furthermore, on the basis of Young's modulus, MWCNT resist tractions one hundred times higher than steel, while weighing six times less [5]. They are also very flexible, and may be bent to angles greater than $90^{\circ}$ without damage. These mechanical features also make MWCNT promising candidates for the reinforcement of polymeric materials. Furthermore, improvements in CNT production which guarantee high purity (>95\%) at low production costs $(<200 € / \mathrm{kg})$ make these materials attractive for industrial applications [2]. The properties of MWCNT are highly dominated by their surface characteristics. Weak van der Waals interactions appear between layers of perfect graphitic surfaces, while stronger hydrogen bridge bonds or even covalent bonds may be formed at defect sites. Therefore, the type of bonding at the MWCNT surface may be tailored by control of the dominating surface properties of the graphitic domains. Furthermore, nitrogen or oxygen atoms at the MWCNT surface may be added as functional groups. These groups, or defects, may be used to anchor and immobilize catalysts on the surface [6]. While an increasing number of defects naturally improve the ability to load greater quantities of catalyst, the conductivity and stability of the MWCNT will unavoidably decrease [5] [7].

Clearly, it is critical to determine the ratio between defect sites and graphitic areas on MWCNT (here represented in the form of a D/G ratio) if one wishes to understand their material properties. To this end, resonance Raman spectroscopy is a promising tool, which is already commonly used to analyze the electronic structure of MWCNT. In particular, the resonance effect may be utilized to enhance signals from graphitic lattice vibrations [8]. The fitting procedure employed in this work focuses on three signals which arise from resonant singlephonon lattice vibrations between $1300 \mathrm{~cm}^{-1}$ and $1700 \mathrm{~cm}^{-1}$. The three bands are referred to the G-band, representative for the pristine graphitic lattice vibration $\mathrm{E}_{2 \mathrm{~g}}(\mathrm{q}=0)$ with no change of the dipole momentum, and the D- and D'-bands for lattice vibrations $E_{2 g}(q \neq 0)$ and $A_{1 g}$, which are induced by structural defects [3]. Molecular vibrations are not considered in this fitting procedure. It is com- 
mon practice to compute the $\mathrm{D} / \mathrm{G}$ ratio by either intensity or peak area in order to elucidate trends in the structural defects of graphitic lattices. In this work, we determine the area of these peaks to calculate the $D^{\star} / G$ ratio, where $D^{\star}$ denotes the sum of D and D'.

We also compare the standard fitting procedure for Raman spectra of soot and related carbonaceous materials proposed by Sadezky, et al. with the present procedure [9]. The procedure of Sadezky, et al. includes several additional physical processes such as combined phonons, molecular vibrations, polarization effects, and Kohn anomalies.

The influence of different pre-treatment on graphitic lattices are also analyzed in terms of their $\mathrm{D}^{\star} / \mathrm{G}$ ratios to gain insight into the resulting material properties. MWCNT were treated in various aqueous media, such as hydrochloric, sulfuric, nitric, and nitrosulfuric acids, as well as in $\mathrm{NO}_{\mathrm{x}}$ containing gas atmospheres. The effects of time and temperature on the nitrosulfuric acid pre-treatment are also investigated.

In this study, we have created an analysis method for MWCNT containing high amounts of graphitic structure based on the evaluation of only singlephonon vibrations in Raman spectra [9]. With this method, it is possible to describe the influence of different pre-treatment procedures, time, and temperature on MWCNT through the calculated $D^{\star} / \mathrm{G}$ ratio. Other physical processes may be resolved from the difference spectra of fit and raw spectra, respectively.

In addition to their bulk structures, the metallic impurities of the tested MWCNT are also determined. Remanent metal catalyst, mainly iron used in MWCNT production, lowers the materials stability during electrochemical usage and can affect later applications [10] [11] [12]. Total reflection X-ray fluorescence (TXRF) measurements, which are cross-checked by gravimetric experiments, are performed to determine the influence of the different aqueous acid pre-treatments on removing this excess iron.

\section{Experimental Part}

MWCNT produced from Shangdong Dazhang Nano Materials Co. were treated with hydrochloric acid, sulfuric acid, nitric acid, nitrosulfuric acid or nitrous gases to modify the surface and create oxygen containing functional groups. Pre-treatments were performed in either aqueous media or in gas-phase. For the aqueous reactions, $10 \mathrm{~g}$ of MWCNT were mixed with $500 \mathrm{ml}$ acid. All mixtures were heated to $105^{\circ} \mathrm{C}$ and stirred with $80 \mathrm{rpm}$ for 18 hours, with the exception of experiments using nitrosulfuric acid, which were stirred for 4 hours. In case of pre-treatment with nitrosulfuric acid the reaction time was reduced compared to other pre-treatments to retain the graphitic structure of the MWCNT. Resulting nitrous gases were neutralized with $3 \mathrm{~mol} / \mathrm{l} \mathrm{NaOH}$ solution. Afterwards, the modified MWCNT were filtered and residuals of acid were washed out over several hours. The MWCNT were dried in a vacuumoven at $80^{\circ} \mathrm{C}$ for 12 hours.

For gas-phase reactions, nitrous gases were generated by a reaction of nitro- 
sulfuric acid ( $\left.50 \mathrm{Vol} \% \mathrm{H}_{2} \mathrm{SO}_{4} \mathrm{c}=96 \% / 50 \mathrm{Vol} \% \mathrm{HNO}_{3} \mathrm{c}=65 \%\right)$ and carbon. The mixture was heated to $120^{\circ} \mathrm{C}$ and the aqueous portion of the resulting vapor was separated via reflux condenser. The dry vapor was lead to a stirred sample containing $7 \mathrm{~g} \mathrm{MWCNT}$. The exposure time was 20 hours at room temperature $\left(\sim 22^{\circ} \mathrm{C}\right)$ and $75^{\circ} \mathrm{C}$.

Raman spectra were recorded using a Thermo Scientific DXR Raman Microscope (Germany) with a 50x magnification and $532 \mathrm{~nm}$ laser. The samples were measured with a $2 \mathrm{~s}$ dwell time and an exposure rate of two. The laser power was $3 \mathrm{~mW}$.

The iron concentration was determined by a S2 Picofox total reflection X-ray fluorescence (TXRF) form Brucker (USA) with molybdenum X-ray tube. $100 \mu \mathrm{l}$ of a $2 \mathrm{mg} / \mathrm{ml}$ stock solution of each MWCNT sample was prepared, mixed with $0.5 \mathrm{ml} 2 \mathrm{~mol} / \mathrm{l}$ nitric acid, and dispersed using an ultrasonic bath. $10 \mu \mathrm{l}$ of the resulting solution was placed on a TXRF object plate and dried in an oven [12]. Every sample was prepared and measured five times to calculate the average and standard distributions. The samples were exposed for $600 \mathrm{~s}$ at $20 \mathrm{keV}$.

The TEM/STEM images were taken by a Hitachi HD-2700 STEM (Japan). The accelerating voltage was $200 \mathrm{kV}$.

\section{Fit Analysis}

For statistical evidence, ten Raman spectra were taken of each sample; measurement points were selected from widely distributed areas over the whole sample via a microscope connected to the Raman setup to guarantee statistical evident data. In this way, it was possible to gain information about the homogeneity of the sample. The spectra were analyzed by the fitting procedure of Sadezky, et al. which was developed for soot and related carbonaceous materials. This method uses five peaks which are representative of the graphitic and amorphous fractions of a given sample; four of these peaks represent the graphitic sites of the samples, and one peak is assigned to the amorphous fraction. Additionally, spectra were analyzed using our fitting procedure, which uses three peaks arising from single-phonon resonances (SPR). These peaks correspond to the G- , D- , and D'-bands. The amorphous fraction of the peak is not regarded in this procedure. A direct comparison of both fitting procedures can be found in Table 1. All peaks in either fitting method, with the exclusion of the amorphous carbon

Table 1. Table type styles (Table caption is indispensable).

\begin{tabular}{crc}
\hline Peak position $\left[\mathrm{cm}^{-1}\right]$ & Sadezky et al. fit & SPR fit \\
\hline 1180 & Disordered graphitic lattice (D4) & Disordered graphitic lattice (D) \\
1360 & Amorphous carbon (A) & Disordered graphitic lattice (D) \\
1500 & Ideal graphitic lattice (G) & Ideal graphitic lattice (G) \\
1580 & Disordered graphitic lattice (D') & Disordered graphitic lattice (D')
\end{tabular}


peak, were fitted using a Lorentzian line shape. The amorphous carbon peak was fitted using a Gaussian line shape, as described by Sadezky, et al. [9].

Reproducibility was verified by a statistical t-test. Each chemical pre-treatment was repeated separately three times at $105^{\circ} \mathrm{C}$ and analyzed independently.

Both fitting procedures were compared using nitric acid functionalized MWCNT. Afterwards, Raman spectra of MWCNT which were functionalized by alternative pre-treatment were compared with one another. The statistical significance between the analyzed samples was also determined via $t$ - and f-tests.

\section{Results and Discussion}

\subsection{Comparison of Fit Procedures}

To investigate the differences of the single-phonon resonance, or SPR, based fitting procedure with that of Sadezky, et al., the same spectrum of nitric acid treated MWCNT was analyzed using each method, as illustrated in Figure 1. In either fitting procedure the D-, G- , and D'-bands are the most dominant features.

The differences between fitted and measured spectra arise from either doubleresonance two phonon processes (around $1100 \mathrm{~cm}^{-1}$ ) or a combination of amorphous carbon, polarization effects, and Kohn anomalies (around $1500 \mathrm{~cm}^{-1}$ ) [13] [14]. As a bench mark, the determined peak areas are compared with those of an untreated MWCNT sample using the aforementioned procedures (Figure 2), with standard deviations in peak areas calculated from repeated measurements. In Figure 2(a), peak areas derived from the approach of Sadezky, et al. are presented, while peak areas derived from the SPR based fit are shown in Figure 2(b).

Spectra fitted by the method of Sadezky, et al. (Figure 1(a)) exhibit smaller deviations from the measured spectra in exactly the regions of 1100 and 1500 $\mathrm{cm}^{-1}$. However, the standard deviations of these peak areas are significantly

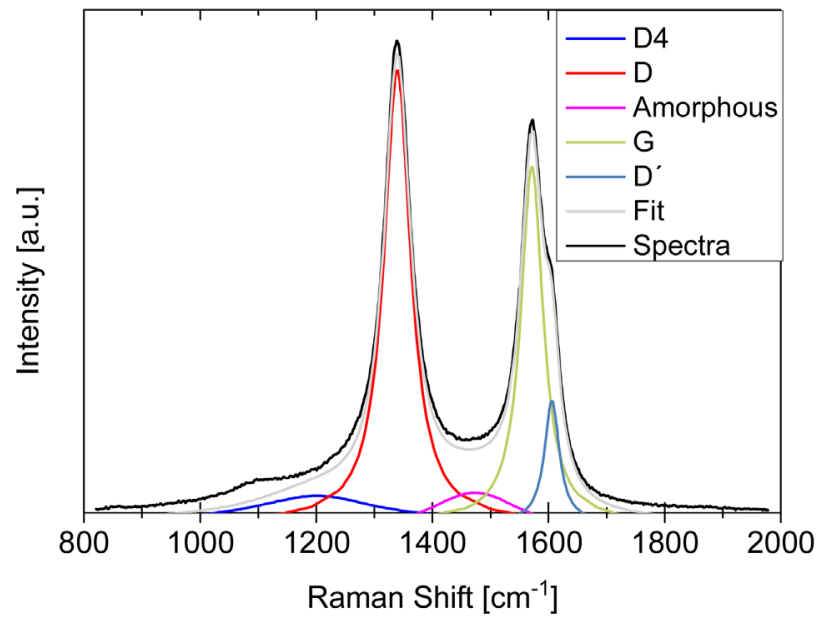

(a)

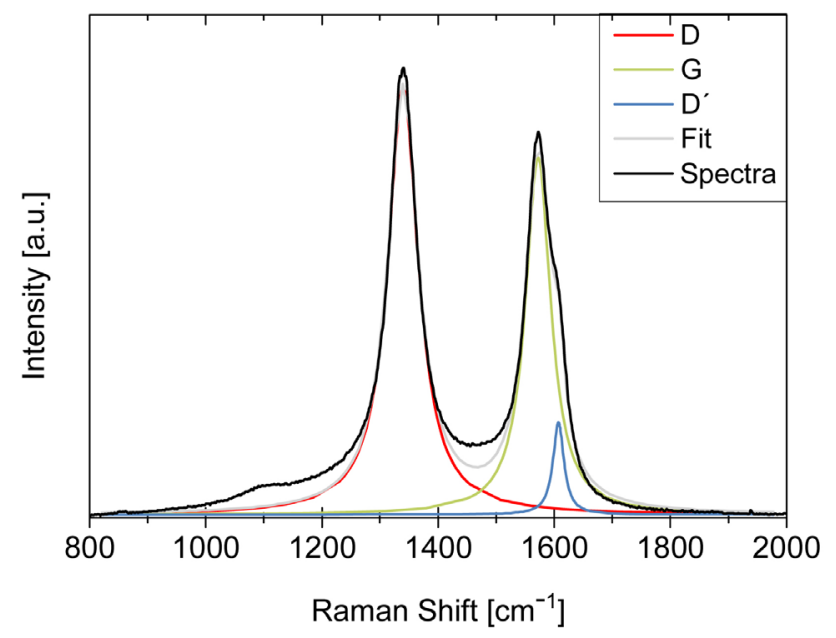

(b)

Figure 1. Raman spectra fitted by Sadezky, et al. procedure (a). Raman spectra fitted by the SPR procedure (b). 


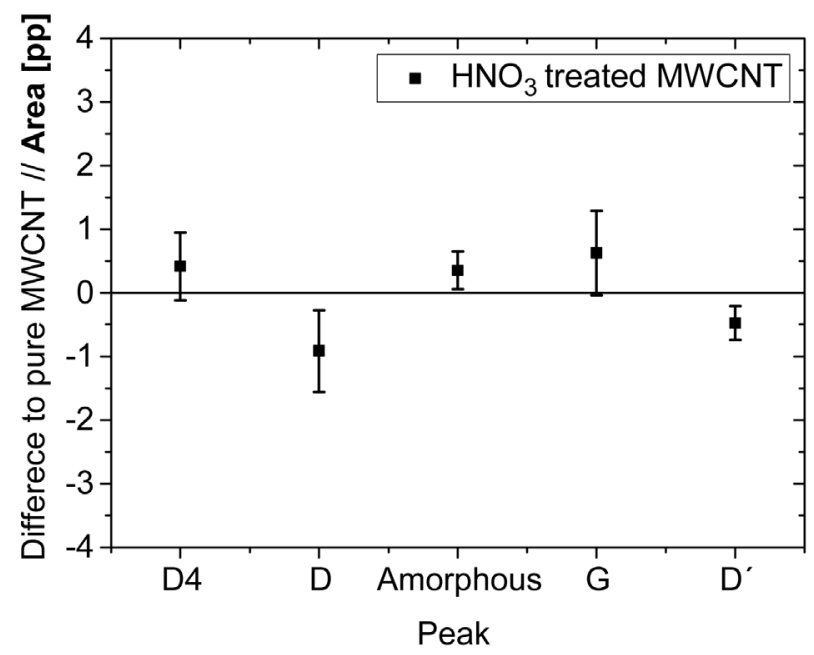

(a)

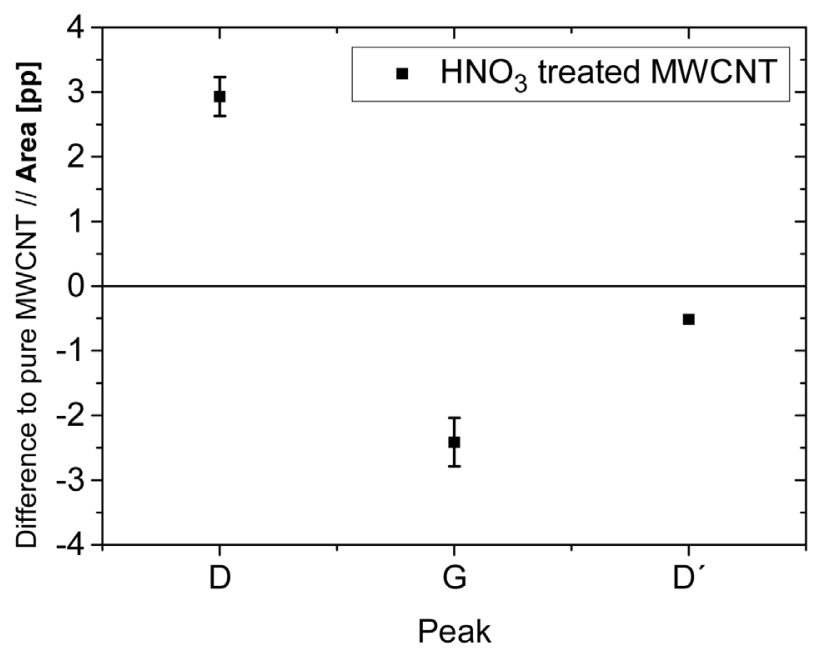

(b)

Figure 2. Difference in fitted peak areas compared to pristine MWCNT. Standard deviations are shown as error bars. Peak areas are derived from Sadezky, et al. procedure (a) and SPR procedure (b).

higher than those of the SPR procedure. The higher standard deviation hinders one's ability to separately analyze differently treated samples as they are less statistically distinguishable. Differences in the standard deviation of the two fitting procedures result from variations in the positions of the D4 and amorphous carbon peaks in the Sadezky procedure; these variations in peak position are presented in Figure 3(a).

The D4 and amorphous carbon peaks are seen to vary strongly as these signals arise from combined processes. Furthermore, the amount of amorphous carbon or other non-graphitic species present in the analyzed MWCNT is quite small, showing only a negligible influence on the spectra. Therefore, the two additional peaks for D4 and amorphous carbon are subject to greater influence from error in the fitting process than the other described physical processes. In samples with higher contents of amorphous carbon, the recorded spectra are even more affected. For these kinds of samples, the fitting route given by Sadezky, et al. is recommended. However, the samples analyzed in the present study exhibit only a small amount of amorphous carbon, and thus the effect of molecular vibrations on the shape of the spectra is also negligible. Since the SPR fitting procedure is able to describe the peak positions of every single-phonon effect (G- , D- , and D'-bands) accurately, it enables a separation of different pre-treated MWCNT due to smaller error in the fitting of peak area. Hence, the SPR procedure ensures higher reproducibility and a better description of the MWCNT sample.

Predictions regarding combined phonon and molecular vibrations, as well as polarization effects and Kohn anomalies, may also be made regarding the difference spectra of the SPR fitted and raw data. Figure 4(a) shows a difference curve between measured and SPR fitted spectra of the nitric acid treated sample congruent with Figure 1(b), additionally differences curves of different pre- 


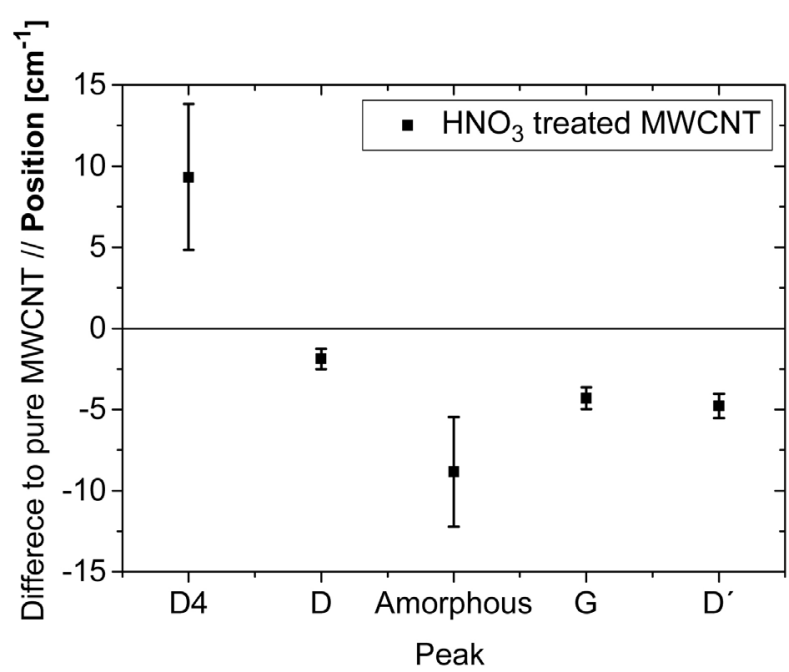

(a)

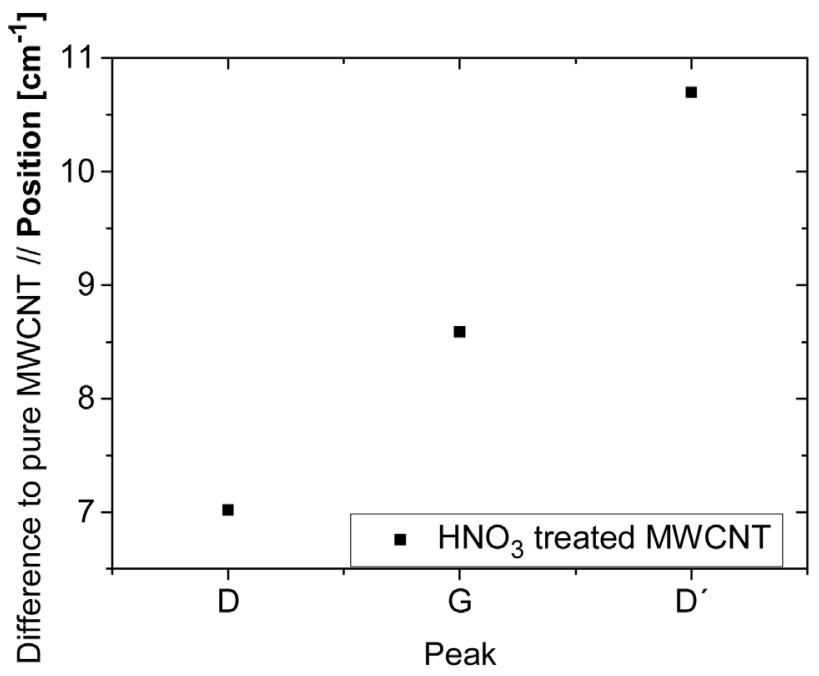

(b)

Figure 3. Shift of peak position in relation to pure MWCNT. Standard deviations are shown as error bar. Fitted by the procedure of Sadezky, et al. (a) and the SPR procedure (b).

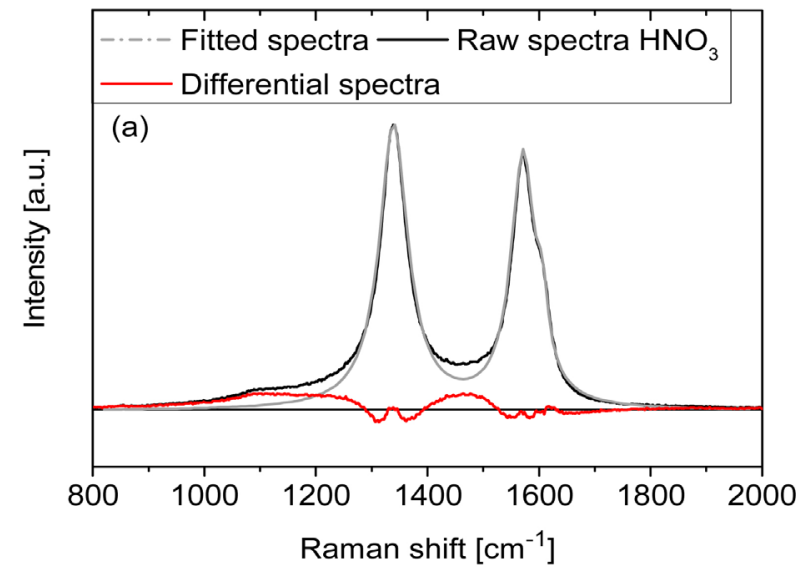

(a)

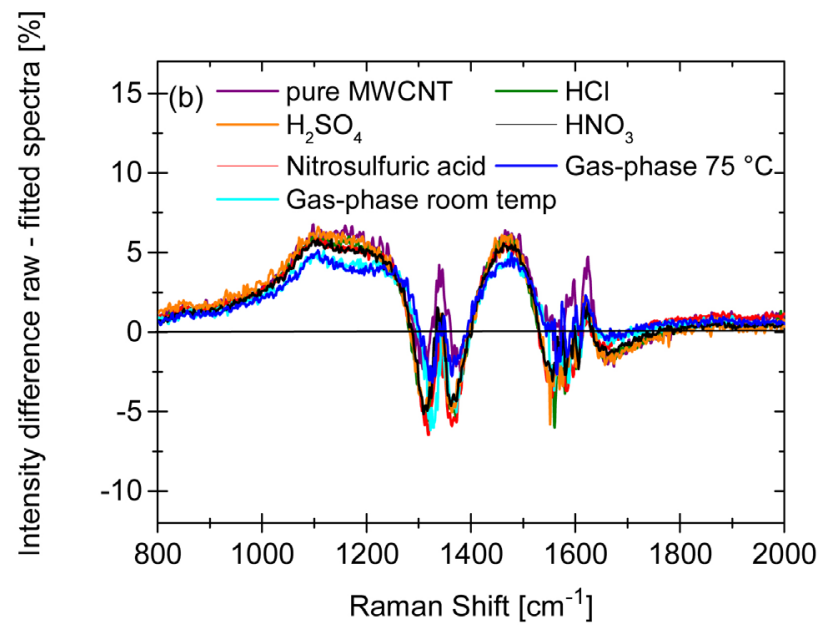

(b)

Figure 4. (a) Raman spectra of nitric acid treated MWCNT. The SPR fitted spectrum (gray) is subtracted from the measured raw spectrum (black), which results in the shown difference spectrum (red). (b) Differential spectra of the fitted and raw spectra for a variety of pre-treated MWCNT.

treated MWCNT are shown in Figure 4(b).

The residuals of the raw and fitted spectra between $1000 \mathrm{~cm}^{-1}$ and $1300 \mathrm{~cm}^{-1}$ are generated by double resonance effects from two phonon vibrations as well as molecular vibrations of alkane chains. The differences between $1400 \mathrm{~cm}^{-1}$ and $1520 \mathrm{~cm}^{-1}$ arise from polarization effects and Kohn anomalies. Molecular vibrations including phenol and carboxyl groups also exist in this energetic region. As amorphous carbon is only present in small quantities in our MWCNT sample, only minor effects on the spectra are seen.

The difference spectra shown in Figure 4(b) exhibit only minor changes between the various pre-treatment procedures employed. Negative signals arise 
from small errors in fitting as Raman peaks have no pure Lorentzian shape and due to the appearance of molecular vibrations, which underlie this resonance area. Whereas, the larger positive differences between fitted and raw peaks around $1000-1250 \mathrm{~cm}^{-1}$ arise from vibrational interactions between molecules in the graphitic lattice, and between $1400-1550 \mathrm{~cm}^{-1}$ from Kohn anomalies and other resonance effects. Due to the similarity of the difference spectra in Figure 4(b), it is suggestive that the impact of pre-treatment cannot be elucidated so easily, and requires a deeper analysis through dominant D-, G- and D'-peaks. Further evaluation of different surface functional groups is impossible as molecular vibrations are overlapped with Kohn anomalies and other resonance effects, creating a complex system of variables.

From this, we have shown that various pre-treatment of MWCNT with a large amount of graphitic structures may be modelled with a higher degree of accuracy using the SPR approach. Other physical processes which may arise in Raman spectroscopy are observed, but remain minimal. The involvement of additional physical processes which overlap energetically with the bands involved in the SPR fitting procedure results in the observed discrepancies in fit peak positions, and also prevent an exact determination of peak areas. Despite this inevitability, the developed SPR procedure, as affected by the phonon interaction within the first Brillouin zone, offers a possibility in more accurately determining relative peak areas.

\subsection{Influence of Pre-Treatment on MWCNT}

With this knowledge in hand, we have applied our SPR method to analyze samples treated by various chemical methods. The Raman spectra of each sample were measured and analyzed following the methods previously described. The significance of changes in spectra arising from different pre-treatment of the MWCNT was verified via t-test.

Figure 5 shows the influence of different pre-treatment on the peak areas of the D-, G-, and D'-bands of MWCNT, respectively. It is seen that pre-treatment with sulfuric and hydrochloric acid has no significant influence on the fraction of the G-peak area (Figure 5(a)). Therefore, a distinction between these methods using the area of the G-band peak is not feasible. A greater change in peak area of the G-band between untreated MWCNT and those pre-treated with nitric acid is observed. A decrease in area of the G-band corresponds to a decrease in the number of graphitic sites relative to the pure MWCNT sample. This decrease is even clearer for samples that are pre-treated with nitrosulfuric acid. Indeed, this is particularly noteworthy since the exposure time of the nitrosulfuric acid pre-treatment is about one fifth that used for the nitric and hydrochloric acid pre-treated samples. The graphitic portion of the MWCNT is further reduced by gas phase pre-treatment with nitrous fumes at both room temperature and at $75^{\circ} \mathrm{C}$, as indicated by the G-bands in Figure 5(a). However, this decrease is also accompanied by an increase in deviation of the determined peak 

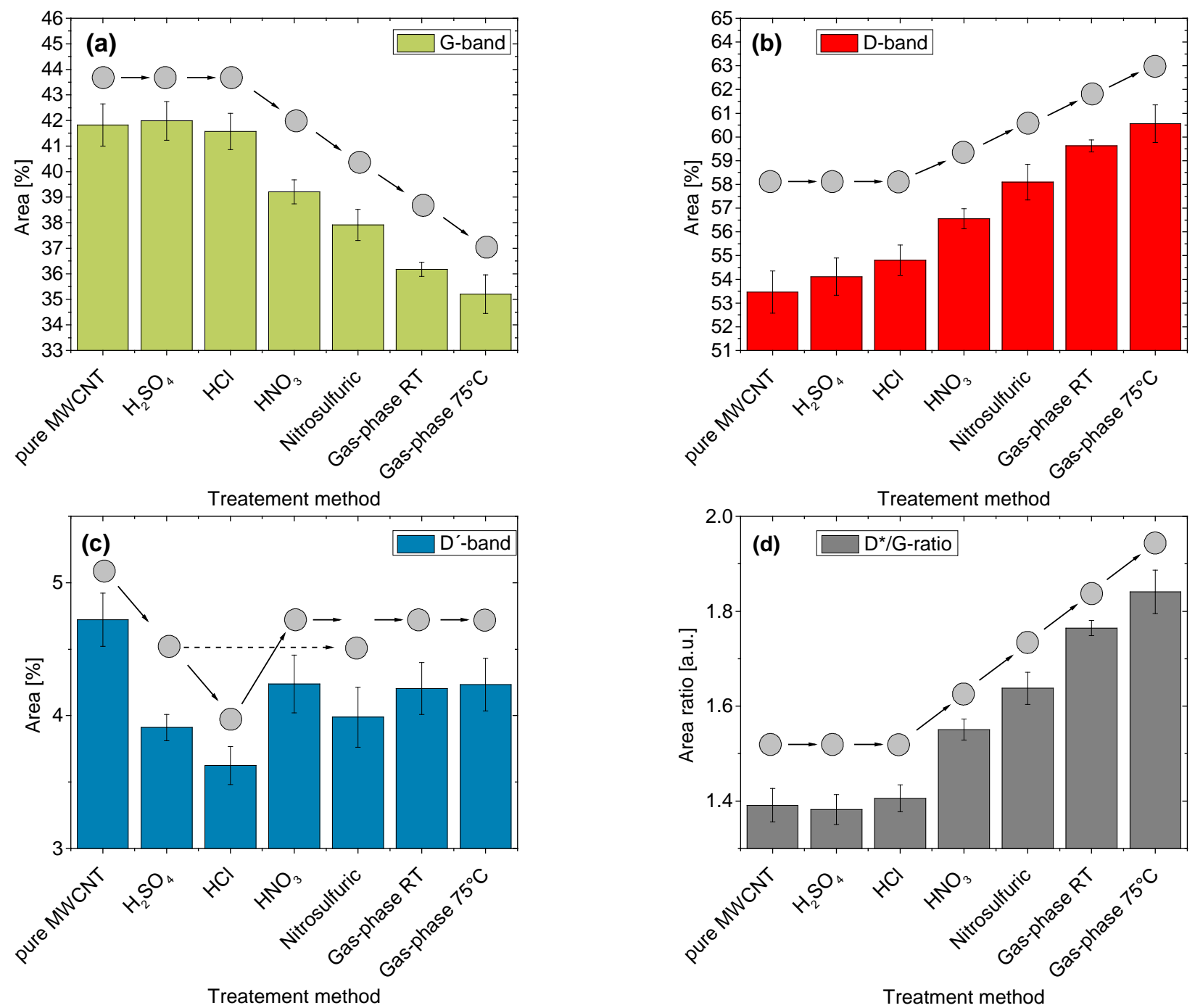

Figure 5. Fractions of the peak areas of the (a) G- , (b) D- and (c) D'-bands for a variety of different acid pre-treated MWCNT. (c) $\mathrm{D}^{\star} / \mathrm{G}$-ratio of different treated samples. $\mathrm{D}^{\star}$ corresponds to the sum of peak areas of $\mathrm{D}$ - and $\mathrm{D}^{\prime}$-Bands. The error bars were calculated from error propagation. The circles over the columns serve to visualize the statistical states of the samples. A horizontal arrow between the circles indicates no significant statistical change between two samples, while a sloping arrow illustrates a statistical significance between the compared samples.

area. This is explained by the lower sample homogeneity induced by this pretreatment. Due to the larger degree of error, it is not possible to statistically justify the observed differences between the gaseous pre-treated samples at room temperature and $75^{\circ} \mathrm{C}$. The smaller errors of the aqueous pre-treatments imply that these methods result in a greater degree of sample homogeneity compared to the gas-phase pre-treatment.

A clear trend is also visible in the fitted D-bands of the differently treated samples, as shown in Figure 5(b). The peak area of the D-band is increased with all acid pre-treatment relative to untreated MWCNT, and as far as 3 percentage points in case of nitric acid. However, differences between the untreated MWCNT and sulfuric and hydrochloric acid pre-treated samples are not large 
enough to establish statistical significance. Meanwhile, gas-phase pre-treatment with nitrous fumes increases the peak area even further, up to 7 percentage points.

Comparing the statistical significance of the D' band between the tested samples, the areas decrease for all acid pre-treated MWCNT (Figure 5(c)).

The $\mathrm{D}^{*} / \mathrm{G}$ ratio is calculated by adding the absolute areas of the $\mathrm{D}$ - and $D^{\prime}$-bands, and dividing by the area of the G-band; the resulting $D^{\star} / G$ ratios are summarized in Figure 5(d). Exclusively taking the area of these three peaks into account leads to significant differences of the applied pre-treatment. In the literature, the $D^{\star} / G$ ratio is often determined by peak intensities, an approach which does not account for the possible superposition of the G- and D'-bands, using intensities is therefore insufficient. This discrepancy is overcome by use of the fit peak areas of either band for the calculation of $D^{*} / G$ ratio

With this approach, the calculated $\mathrm{D}^{*} / \mathrm{G}$-ratios show an increase in the relative number of graphitic structural defects with increasing oxidative character of the acids used for pre-treatment. In detail, pre-treatment with sulfuric or hydrochloric acid do not induce significant changes in the $\mathrm{D}^{*} / \mathrm{G}$ ratio, while an increase of about $12 \%$ is observed when using nitric or $17 \%$ if nitrosulfuric acid is used. Even higher $D^{\star} / G$ ratios (up to $32 \%$ compared to untreated MWCNT) can be reached by gas-phase pre-treatment at different temperatures.

Electron microscopy imaging supports the trends observed by Raman spectroscopy. STEM images (Figures 6(a)-(d)) illustrate the surface roughness, which increases in order of sulfuric acid $<$ nitric acid $<$ nitrosulfuric acid $<$ nitrous fume gas-phase pre-treatment. This roughness is accompanied with an increase in the number of defects and correspondingly a decrease in graphitic structure. TEM images reveal defects at the surface layers of the MWCNT by pre-treatment with sulfuric acid, nitric acid and in gas-phase, as shown in Figure 6(g), Figure 6(h), and Figure 6(j), respectively. Nitrosulfuric acid decomposes

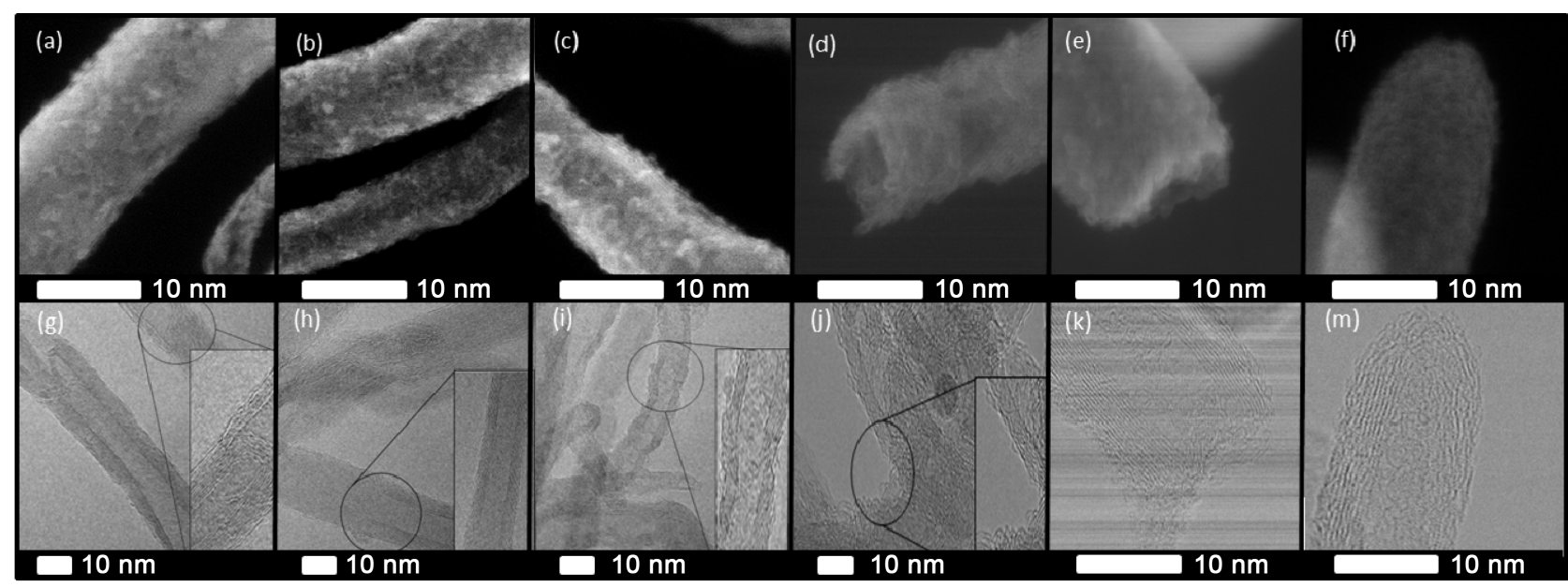

Figure 6. The upper row shows STEM images of MWCNT, while in the lower row TEM images are presented. (a)/(g) Sulfuric acid treated, (b)/(h) Nitric acid, (c)/(i) Nitrosulfuric acid, (d)/(j) NOx gas-phase pre-treatment, (e)/(k) Open endcap of a nitrosulfiric acid treated sample, (f)/(l) and closed endcaps of a gas-phase treated sample. 
not only surface layers of the MWCNT, but also penetrates deeper, as seen by the discontinuity of the graphene layers in Figure 6(i). The surfaces of the aqueous treated MWCNT are nearly free of amorphous carbon (Figures 6(g)(i)), whereas the gas-phase treated sample shows some residual (Figure 6(j)). All observations made by microscopy are in perfect agreement with the results of the Raman analysis.

To gain deeper insight into the kinetics of defect formation during the functionalization process, varying temperatures and pre-treatment times were investigated. These studies were performed solely for the nitrosulfuric acid pretreatment due to its high rate of defect formation and good homogeneity compared with other pre-treatment used. Four different temperatures $\left(55^{\circ} \mathrm{C}, 75^{\circ} \mathrm{C}\right.$, $95^{\circ} \mathrm{C}$ and $110^{\circ} \mathrm{C}$ ) were applied over a four hour reaction time. Raman analysis was subsequently used to determine the progression of defect formation (Figure 7). The amount of defect formation increases as a function of both reaction time and temperature, while the amount of graphitic structure decreases. The standard deviation of samples treated for longer times also decreases, which indicates greater sample homogeneity. The reaction temperature itself has a substantial influence on the initial formation of defects, as seen by the D- and G-band areas acquired after 60 minutes. A subsequent change of up to 2 - 3 percentage points in the D- or G-band areas was also determined independent of the initial defect ratio. This means that the majority of defect formation occurs within the first 60 minutes of the reaction time.

\subsection{Influence of Pre-Treatment on Iron Contamination in MWCNT}

The influence of iron contamination on the MWCNT following acid pre-treatment was determined by either total reflection $\mathrm{x}$-ray fluorescence (TXRF) or gravimetric analysis.

Pre-treatment with sulfuric acid removed approximately $50 \%$ of iron conta-

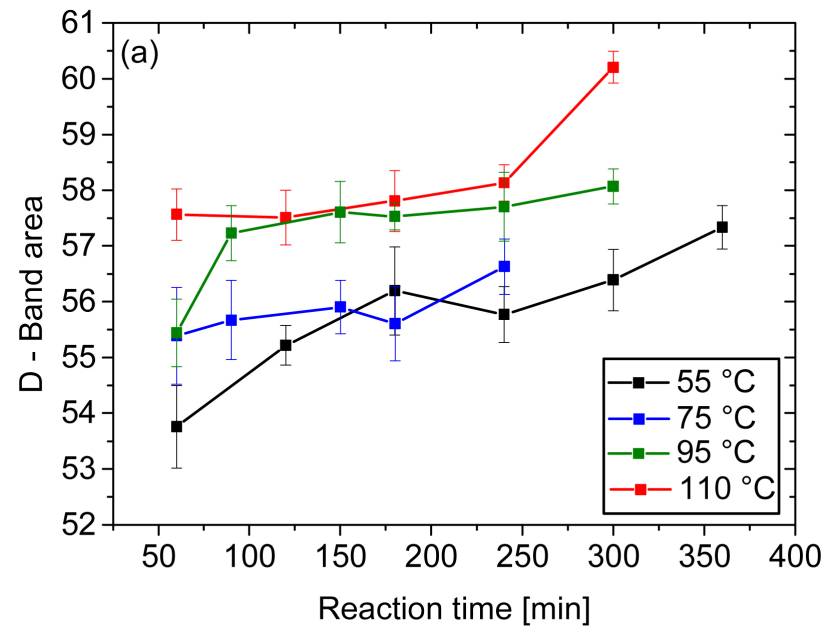

(a)

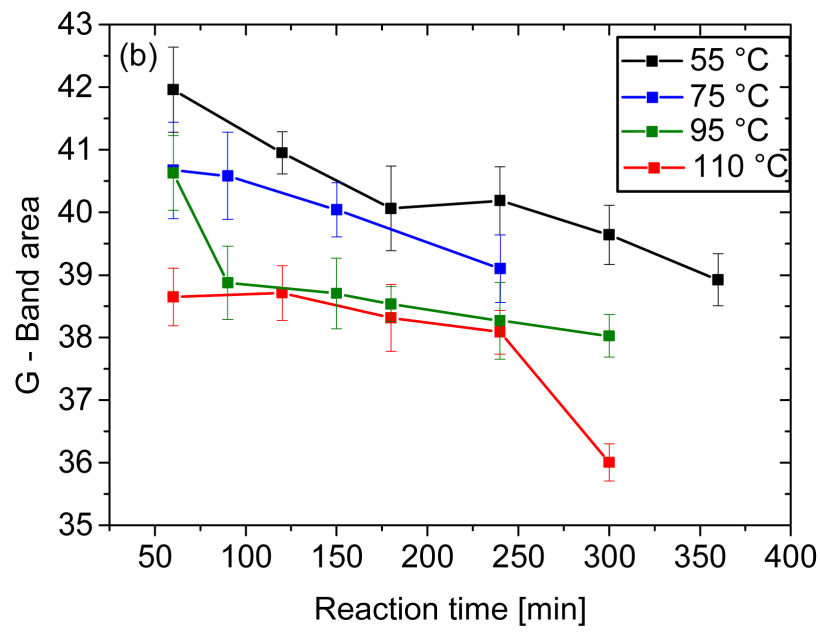

(b)

Figure 7. Progression of nitrosulfuric acid treated samples at different temperatures (a) D-Band (b) G-Band. 
mination compared to pure MWCNT (Figure 8). Decreases in iron contamination by $60 \%$ and $65 \%$ in the respective $\mathrm{HCl}$ and nitric acid pre-treated samples were also found. Nitrosulfuric acid was also found to be the most efficient pre-treatment, with only a small fraction of $0.05 \mathrm{wt} \%$ iron determined by TXRF and thus effectively decreasing the iron content by a factor of $\times 10$. This observation can be explained by the opening of the ends of the MWCNT, which has been previously reported [15]. This allows for encapsulated iron to also be washed out from the inner portion of the MWCNT. These trends are observed also by microscopy, as seen in Figure 6(e), Figure 6(k); this is in contrast to gas-phase treated MWCNT, in which endcaps remain closed (Figure 6(f), Figure $6(\mathrm{~m})$ ). While acid pre-treated samples no longer exhibited observable surface iron, samples treated by gas-phase functionalization still contain iron particles at their surface (see Figure 6(j)). The amount of iron determined by TXRF was confirmed by gravimetric analysis of the used acids for the pre-treatment, as depicted in Figure 8 (red bar). After neutralization, the precipitated iron hydroxide was oxidized to $\mathrm{Fe}_{2} \mathrm{O}_{3}$ in an oven at $800^{\circ} \mathrm{C}$ until the weight was constant [16]. The residual amount of iron contamination after the pre-treatment with hydrochloric acid was calculated based on the gravimetric measurement. The total amount of iron determined by TXRF and gravimetric measurements correspond to the amount of iron in untreated MWCNT. Determination of the iron content by gravimetric analysis was not possible for sulfuric acid treated samples as the quantity of precipitated iron hydroxide was below our detection limit. During TXRF measurements, matrix effects like graphitic coated iron or sulfate residuals do not affect the results. Disintegration is also unnecessary [12].

\section{Conclusion}

A fitting procedure based on single-phonon resonances for the Raman spectra of MWCNT has provided us the opportunity to gain detailed and statistically sig-

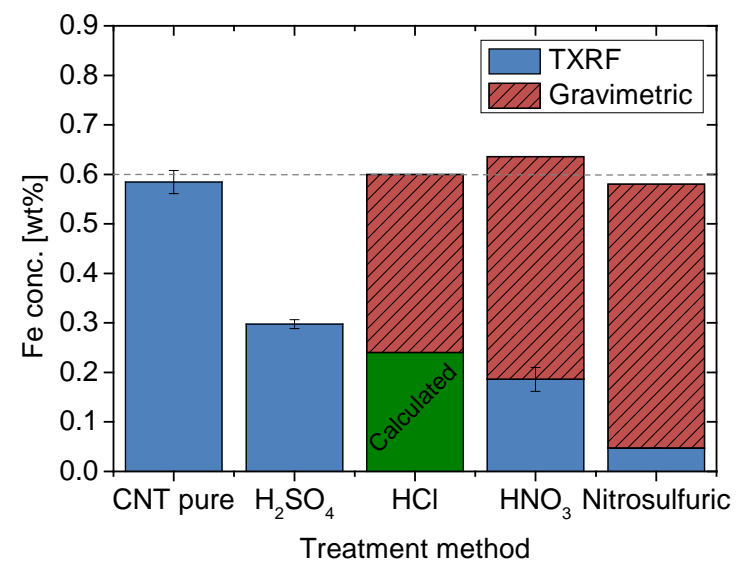

Figure 8. Iron concentrations of different pre-treated MWCNT. Residual amount of iron contamination in MWCNT measured by TXRF (blue bar), amount of washed out iron during the acid pretreatment determined by gravimetry (red bar). 
nificant information regarding the D-, G-, and D'-bands of samples with relatively low quantities of non-graphitic structures. It is important to note, however, that this fitting procedure is not universally applicable for carbon materials. Samples with a high content of amorphous carbon should be analyzed by the fitting approach of Sadezky, et al. [9], whereas the present fitting procedure is recommended for samples with high graphitic structure content. We have found that the SPR approach to analyze Raman spectra of MWCNT is particularly sensitive to differences arising in the various employed pre-treatments, while other physical processes which arise in Raman spectroscopy are minimally affected. As a result, the SPR procedure provides a promising method of investigating significant changes in the bulk composition of MWCNT which has undergone a variety of pre-treatments. This has also allowed for the determination of relative sample homogeneity by comparison of the statistical error produced by a given pre-treatment technique. A general decrease in error was made possible through the SPR procedure in the form of reduced peak shifting. The influences of reaction time and temperature on defect formation during nitrosulfuric acid pre-treatment were also evaluated, revealing that the number of structural defects increases continuously with reaction time. The reaction temperature has a large impact on the rate of defect formation during the first 60 minutes. Afterward this initial time, the rate of defect formation is comparable for all investigated temperatures. Slower rates of defect formation can be compensated by using longer reaction times, which also leads to a greater homogeneity of the sample. STEM and TEM images confirm our conclusions derived from Raman spectroscopy. The remaining iron contamination remaining from catalyst during the synthesis process is also influenced by the choice of acid pre-treatment, with the nitrosulfuric acid pre-treatment appearing the most efficient. With these results, it is possible to predict the degree of defect formation and homogeneity of a MWCNT sample, allowing for the preparation of MWCNT with reproducible bulk properties.

\section{Acknowledgements}

The author would like to thank Prof. Dr. Alexander Auer and Dr. DimitriosManganas for their support by theoretical chemical questions. Furthermore, we would like to thank Prof. Dr. Lehman form the Max Planck Institute for coal research to provide the STEM and Prof. Dr. Schram from the Hochschule Niederrhein, University of Applied Science to provide the TXRF. Also thanks to Birgit Deckers for the support in graphic design.

\section{References}

[1] Jorio, A. (2008) Carbon Nanotubes: Advanced Topics in the Synthesis, Structure, Properties and Applications. In: Tomanek, D., Ed., Introduction to the Important and Exciting Aspects of Carbon-Nanotube Science and Technology, Springer, Heidelberg, 1-12. https://doi.org/10.1201/NOE0849396397.ch61

[2] Tessonnier, J.P., Rosenthal, D., Hansen, T.W, Hess, C., Schuster, M.E., Blume, R., 
Girgsdies, F., Pfaender, N., Timpe, O., Su, D.S. and Schloegl, R. (2009) Analysis of the Structure and Chemical Properties of Some Commercial Carbon Nanostructures. Carbon, 47, 1779-1798. https://doi.org/10.1016/j.carbon.2009.02.032

[3] Jorio, A. (2012) Raman Spectroscopy in Graphene-Based Systems: Prototypes for Nanoscience and Nanometrology. ISRN Nanotechnology, 2012, 1-16. https://doi.org/10.5402/2012/234216

[4] Aqel, A.A.E., El-Nour, K.M.M., Ammar, R.A.A. and Al-Warthan, A. (2012) Carbon Nanotubes, Science and Technology Part (I) Structure, Synthesis and Characterisation. Arabian Journal of Chemistry, 5, 1-23. https://doi.org/10.1016/j.arabjc.2010.08.022

[5] Treacy, M.M.J., Ebbesen, T.W. and Gibson, J.M. (1996) Exceptionally High Young's Modulus Observed for Individual Carbon Nanotubes. Nature, 381, 678-680.

https://doi.org/10.1038/381678a0

[6] Pinna, N.M.K. (2012) Atomic Layer Deposition of Nanostructured Materials. In: Musgrave, C.B., Ed., Theoretical Modeling of ALD Processes, Wiley-VCH, Weinheim, 3-22.

[7] Serp, P., Corrias, M. and Kalck, P. (2003) Carbon Nanotubes and Nanofibers in Catalysis. Applied Catalysis A, 253, 337-358. https://doi.org/10.1016/S0926-860X(03)00549-0

[8] Jorio, A. (2013) Raman Spectroscopy in Graphene Related Systems. In: Jorio, A., Ed., Vibrations in SP2 Nanocarbons, Wiley-VCH, Weinheim, 53-69.

[9] Sadezky, A., Muckenhuber, H., Grothe, H., Niessner, R. and Poschl, U. (2005) Raman Microspectroscopy of Soot and Related Carbonaceous Materials: Spectral Analysis and Structural Information. Carbon, 43, 1731-1742. https://doi.org/10.1016/j.carbon.2005.02.018

[10] Frank, B., Rinaldi, A., Blume, R., Schloegl, R. and Su, D.S. (2010) Oxidation Stability of Multiwalled Carbon Nanotubes for Catalytic Applications. Chemistry of Materials, 22, 4462-4470. https://doi.org/10.1021/cm101234d

[11] Kocot, K., Zawisza, B., Margui, E., Queralt, I., Hidalgo, M. and Sitko, R. (2013) Dispersive Micro Solid-Phase Extraction Using Multiwalled Carbon Nanotubes Combined with Portable Total-Reflection X-Ray Fluorescence Spectrometry for the Determination of Trace Amounts of $\mathrm{Pb}$ and $\mathrm{Cd}$ in Water Samples. Journal of Analytical Atomic Spectrometry, 28, 736-742. https://doi.org/10.1039/c3ja50047k

[12] Margui, E., Zawisza, B. and Sitko, R. (2014) Trace and Ultratrace Analysis of Liquid Samples by X-Ray Fluorescence Spectrometry. Trends in Analytical Chemistry, 53, 73-83. https://doi.org/10.1016/j.trac.2013.09.009

[13] Dresselhaus, M.S., Jorio, A. and Saito, R. (2010) Perspectives on Carbon Nanotubes and Graphene Raman Spectroscopy. Annual Review of Condensed Matter Physics, 1, 89-108. https://doi.org/10.1146/annurev-conmatphys-070909-103919

[14] Thomsen, C., Reich, S. and Maultzsch, J. (2004) Resonant Raman Spectroscopy of Nanotubes. Philosophical Transactions of the Royal Society of London Series AMathematical Physical and Engineering Sciences, 362, 2337-2359. https://doi.org/10.1098/rsta.2004.1444

[15] Solhy, A., Machado, B.F., Beausoleil, J., Kihn, Y., Gonçalves, F., Pereira, M.F.R., Órfão, J.J.M., Figueiredo, J.L., Faria, J.L. and Serp, P. (2008) MWCNT Activation and Its Influence on the Catalytic Performance of Pt/MWCNT Catalysts for Selective Hydrogenation. Carbon, 46, 1194-1207. https://doi.org/10.1016/j.carbon.2008.04.018

[16] Jander, G. (1989) Maßanalyse. In: Jander, G., Ed., Gravimetrie, De Gruyter, Berlin, 145. 
Submit or recommend next manuscript to SCIRP and we will provide best service for you:

Accepting pre-submission inquiries through Email, Facebook, LinkedIn, Twitter, etc. A wide selection of journals (inclusive of 9 subjects, more than 200 journals)

Providing 24-hour high-quality service

User-friendly online submission system

Fair and swift peer-review system

Efficient typesetting and proofreading procedure

Display of the result of downloads and visits, as well as the number of cited articles Maximum dissemination of your research work

Submit your manuscript at: http://papersubmission.scirp.org/

Or contact mas@scirp.org 